\title{
Du brouillage. Droite et gauche en Martinique
}

Quelle réalité?

Interference. Right and left wings in Martinique. And the reality?

Jean-Claude William

\section{(2) OpenEdition}

Journals

Édition électronique

URL : http://journals.openedition.org/plc/173

DOI : $10.4000 /$ plc. 173

ISSN : 2117-5209

Éditeur

L'Harmattan

Édition imprimée

Date de publication : 15 décembre 2007

Pagination : 121-149

ISBN : 2-296-01986-2

ISSN : $1279-8657$

Référence électronique

Jean-Claude William, « Du brouillage. Droite et gauche en Martinique », Pouvoirs dans la Caraibe [En

ligne], 15 | 2007, mis en ligne le 06 mai 2011, consulté le 01 mai 2019. URL : http://

journals.openedition.org/plc/173; DOI : 10.4000/plc.173 


\title{
DU BROUILLAGE. DROITE ET GAUCHE EN MARTINIQUE. QUELLE REALITE ?
}

\author{
Jean-Claude WILLIAM \\ Professeur de science politique \\ Membre du CRPLC \\ Université des Antilles et de la Guyane
}

Dans les démocraties libérales, on s'accorde souvent pour considérer que le clivage droite/ gauche a perdu de sa pertinence, l'économie de marché ne pouvant plus «fonctionner » à l'état pur et le plan ayant largement vécu. Cette approche n'implique pas que toute différence a disparu entre ces deux composantes du spectre politique. Beaucoup d'analystes voient dans la culture, l'éducation des domaines où les différenciations idéologiques et politiques se manifestent. Surtout, s'agissant de la France, la question des trente cinq heures, celle des nouveaux contrats en droit du travail soulignent que droite et gauche se distinguent toujours. Ne perdons pas de vue non plus la position par rapport à l'accueil et au séjour des étrangers. Il s'agit dans ce dernier cas, il est vrai, davantage de pratique que d'option théorique.

Les lignes ont bougé, les oppositions sont moins accentuées qu'il y a quelques années du fait en particulier de la mondialisation libérale. Mais elles subsistent.

Le cas de la Martinique, département français ultrapériphérique s'inscrit dans ce contexte. Cependant pour essayer de comprendre la nature du clivage droite/gauche dans une société que nous considérons comme atypique, il convient d'intégrer, nous semble-t-il, des facteurs qui lui sont propres. La Martinique - tel est notre postulat - est une société de brouillage et d'apparence. Elle se caractérise par le rapport dialectique de la pulsion mimétique et de l'affirmation de différence qui constituent les deux figures du Désir de reconnaissance ${ }^{1}$. Pulsion mimétique et affirmation de différence s'entremêlent à tel point qu'une démarche mimétique peut masquer la volonté de différence. Inversement, une conduite de différence peut dissimuler l'aspiration mimétique. Brouillage. La pulsion mimétique a pris la forme de la revendication inlassable de l'assimilation dont la traduction juridico-politique était le statut départemental. L'affirmation de

\footnotetext{
1 Edouard Glissant (Le discours antillais. Paris: Le Seuil, 1981) parle de «pulsion mimétique » pour rendre compte de cette «poussée », au sens freudien du terme, qui a longtemps conduit le Martiniquais à chercher l'identification au Blanc.
} 
l'altérité débouche, elle, sur l'espoir de voir reconnue la différence essentielle entre la Martinique et sa métropole. Pointent, alors, l'idée de nation et la recherche de la souveraineté.

La difficulté vient de ce que l'apparence est parfois trompeuse. Il arrive qu'une revendication en cache une autre. Et des oppositions flagrantes peuvent recouvrir des convergences incontestables.

Les organisations politiques en offrent une bonne illustration. Elles apparaissent - comme en Guadeloupe - sitôt l'avènement de la $\mathrm{III}^{\mathrm{e}}$ République et sont rattachées aux principales organisations métropolitaines. On trouve, en effet, des sections du Parti Radical, du Parti Socialiste et du Parti Communiste ${ }^{2}$. Leurs positions sont relayées par une presse dynamique et volontiers polémique. Ces organisations ont un point commun, l'obtention du statut départemental, consécration de la «Longue Marche » vers l'assimilation. Dès le 24 novembre 1874, le Conseil général de la Martinique émet un vœu en ce sens. Il met l'accent sur le fait que les Martiniquais, les Guadeloupéens sont «Français de droits, de langage, de coutume, de cœur». A l'occasion d'une conférence prononcée en 1935, un médecin, le Docteur Pidery traduit ce sentiment: «Renouvelant l'exemple des Gaulois qui eurent la sagesse de s'incliner devant la culture romaine, de s'en accommoder, de s'en pénétrer pour faire à leur tour de leur nation une grande nation, nos pères ont tout de suite reconnu que la civilisation qui n'avait rien à voir avec leur oppresseur les invitait à la plus haute des destinées, ils l'ont acceptée d'enthousiasme et l'ont assimilée avec la foi de ceux qui savent comprendre et aimer $»^{3}$.

Les parlementaires antillais vont s'associer au mouvement initié par les conseillers généraux et, à intervalles réguliers, ils vont déposer des propositions de loi tendant à l'érection de la Martinique - à laquelle on a joint les autres « vieilles colonies » - en département français ${ }^{4}$. Les Francs-Maçons dont l'influence morale a rapidement été réelle et dont l'implication politique est comparable à ce qu'elle est en France à l'époque prennent aussi position.

\footnotetext{
${ }^{2}$ Voir : - Terrée (sans indication de prénom) «L'élargissement de la vie politique ». Historial Antillais, vol .IV, p.329 et suiv. ; Cécile Celma «La vie politique à la Martinique des années 1910 à 1939 ». Historial Antillais, vol .V p. 318 et suiv.

${ }^{3}$ Cité par A. Blérald. Etat, idéologies et développement à la Guadeloupe et à la Martinique. De l'esclavage au capitalisme. De la ségrégation à l'assimilation, Thèse de Science Politique, dact. , Paris I, p. 389.

${ }^{4}$ Pour une chronologie des initiatives prises en ce sens, nous prenons la liberté de renvoyer à notre article « Les origines de la loi de départementalisation », Historial Antillais, vol. VI, p. 50 et suiv.
} 
Ainsi, réunis en Congrès (toutes obédiences confondues) le 20 novembre 1925, ils reprochent aux gouverneurs d'appliquer les méthodes en usage dans les nouvelles colonies «encore peu évoluées $»^{5}$ et refusent que la Martinique soit soumise au Droit commun colonial.

La loi de 1946 accordant aux «quatre vieilles colonies » - et à elles seules - le statut de département exauce, donc, le vieux rêve d'égalité totale. Du point de vue de la pulsion mimétique, l'objectif est atteint. Mais s'agissant de l'égalité dans le domaine social en particulier, le nouveau statut sera vécu par beaucoup comme une victoire à la Pyrrhus. Trois ans après le vote de la loi, Aimé Césaire déclare : "Quel est le grand fait qui domine la politique martiniquaise depuis 1946 ? C'est le refus du gouvernement français de traiter la Martinique en département français et sa volonté de continuer à nous imposer un régime fondé sur le colonialisme... Si, contrairement à l'esprit de la Constitution, le gouvernement continue à nous imposer un régime d'exception fondé sur le racisme et la discrimination, autrement dit si on nous refuse tous les avantages sociaux, obligation sera faite au peuple martiniquais de donner une autre direction à ses aspirations $»^{6}$.

Convaincue que les Martiniquais ont été abusés, la gauche va se constituer en organisations proprement martiniquaises et revendiquer un nouveau statut. Ici intervient le brouillage. Désormais, la position par rapport au statut va déterminer l'appartenance à la gauche ou à la droite. Est réputé de droite, le partisan du statu quo, est tenu pour un homme de gauche le champion d'un changement de statut. Même si le premier professe sur la question économique et sociale ou les problèmes internationaux des positions progressistes et le second est, dans ces domaines, d'un conservatisme affiché. Plus précisément, l'homme de droite est «assimilationniste », l'homme de gauche, «nationaliste». Mais le temps fait son œuvre. La situation martiniquaise va évoluer, le contexte international également. Le brouillage va de nouveau se manifester. Les ci-devant assimilationnistes en tout cas nombre d'entre eux, vont adopter une démarche identitaire et les nationalistes, tout au moins ceux qui les premiers se sont définis comme tels, n'ont plus en vue

\footnotetext{
${ }^{5} \mathrm{~V}$. Sable. La transformation des isles d'Amérique en départements français. Paris : Larose, 1955 cite des extraits de cette motion (p.135).

${ }^{6}$ «Discours à la Mairie de Fort-de-France». 22 septembre 1949 in N. Nicolas. L'opinion publique martiniquaise devant la loi d'assimilation de 1946. Mémoire pour la maitrise d'Histore. Paris I. 1973, p.85.
} 
l'indépendance même à terme et se situent, eux aussi, dans la problématique de l'affirmation identitaire. Curieuse convergence !

Après avoir essayé de comprendre par quels processus elle s'est produite en considérant d'une part la situation à gauche et à l'extrême gauche (I) et d'autre part à droite (II), nous prendrons le risque de proposer des scénarios pour le futur (III).

\section{DU NATIONALISME A LA REVENDICATION IDENTITAIRE. OUBLI ET REFERENCE FORMELLE}

Le Parti Progressiste Martiniquais (PPM) d'Aimé Césaire tout en se réclamant de l'autonomie se définit comme un parti nationaliste même s'il adopte une démarche contournée et que, dans la dernière période, il ne faisait plus référence au nationalisme. Le Parti Communiste Martiniquais, aujourd'hui très affaibli - c'est une litote - s'est défini de la même manière. Quant aux autres formations dites de gauche, il convient de distinguer. Il y a d'une part, la Fédération Socialiste de la Martinique qui bien qu'étant une Fédération comme les autres du Parti Socialiste affirme son «identité martiniquaise ${ }^{7}$ et d'autre part, un groupe étrange, un OPMI - un Objet Politique Mal Identifié - composé de conseillers généraux qui se laissent appeler les «Divers Gauche » dont la position sur la question identitaire n'est pas d'une clarté totale ${ }^{8}$.

L'extrême gauche dont l'organisation dominante est le Mouvement Indépendantiste Martiniquais exprime sans ambiguïté son nationalisme et sa volonté d'obtenir l'indépendance. Il n'est, cependant, pas interdit de se demander si on n'est pas là dans le domaine de l'incantation.

Pour ce qu'il est convenu d'appeler la gauche, nous braquerons le projecteur sur le PPM (A) et pour ce qui est de l'extrême gauche, nous porterons notre attention sur le MIM (B).

\footnotetext{
${ }^{7}$ Aux élections législatives de mars 1985, son slogan est : «Identité martiniquaise, nationalité française ». France-Antilles. 14 mars 1985.

${ }^{8}$ Il faut aussi mentionner «Bâtir le pays Martinique » dont l'influence est très marquée au Lamentin et dont le chef de file est le maire de cette ville mais qui est également représenté au Conseil régional et qui revendique la souveraineté.
} 


\section{A - LE PPM. DU DISCOURS A LA PRATIQUE}

Le positionnement nationaliste du PPM n'est pas la conséquence de la prise en compte d'une situation objective. Il relève plus du domaine de l'affect que de l'analyse politique. Il repose sur la déception et l'engagement dans cette voie se fait avec réticence, à contre-cœur, gardant longtemps l'espoir que la rupture n'est pas inéluctable. Ce n'est que progressivement que le caractère nationaliste du Parti s'affiche (1). L'autonomie censée être l'enveloppe juridique et politique de la nation a un contenu à géométrie variable (2). L'arrivée de la gauche au Pouvoir en France, en 1981, conduit le PPM à décréter un "moratoire » sur la question du statut. La ligne est pour le moins floue. Le XVII ${ }^{\mathrm{e}}$ Congrès - dont la première partie s'est tenue les 10,11 et 12 juin 2005 - le reconnaît honnêtement: "Nous devons convenir que les positions du Parti n'ont pas été claires ces dernières années ${ }^{9}$. Pour l'heure, en prenant en compte la politique préconisée par le PPM depuis 1981 force est de constater que la revendication est celle de la reconnaissance de l'identité propre du peuple martiniquais et qu'il n'est plus question d'une nation se situant dans la problématique de la souveraineté (3).

\section{AUX SOURCES : LE DEPIT AMOUREUX}

En 1956, avant donc la création du PPM ${ }^{10}$, Aimé Césaire indique avec plus de force encore ce qu'il répète régulièrement depuis 1949. Il affirme, en effet, que c'est l'histoire des dix années de départementalisation qui a fait sortir des limbes ce qu'il appelle le sentiment national : «Je soutiens que si aujourd'hui nous assistons à l'éveil d'un sentiment national aux Antilles françaises c'est à la loi du 19 mars 1946 que nous le devons et que c'est la dialectique elle-même qui donne aux problèmes posés par la départementalisation, la seule issue qui leur puisse convenir: une issue

\footnotetext{
${ }^{9}$ Ce n'est pas la première fois que le PPM se livre à une autocritique publique. Lors du X anniversaire du Parti, le 23 mars 1968, Camille Darsières, membre du Bureau Politique a déclaré : «Le courage aujourd'hui (...) c'est de constater que le Parti Progressiste Martiniquais, en ses premières années, se voyant nécessaire à la vie politique du pays, se paya dangereusement le luxe de laisser planer autour de lui une équivoque. Equivoque d'autant plus pernicieuse que si le PPM, confiant et insouciant ne s'est point occupé à la dissiper, par contre, d'autres sinon tous les autres, prirent peine et plaisir, et trouvèrent intérêt à l'installer et à l'étaler ». Brochure éditée par le PPM sans indication de date ni de lieu.

${ }^{10}$ Il sera créé le 23 mars 1958.
} 
nationale ${ }^{11}$. C'est donc l'échec de la départementalisation qui a conduit à la prise de conscience nationale. En 1961, dans un article publié par Le Monde, il insiste : "Aussi bien la "révolution » antillaise est-elle déjà commencée. Des peuples frustrés du droit de se gouverner eux-mêmes, frustrés du pouvoir d'orienter, fût-ce dans une mesure infime, leur propre destin : à la limite, frustrés d'eux-mêmes, se réveillent à une revendication nouvelle : celle de leur personnalité et de l'autogestion $»^{12}$. Pour l'extérieur - la France-la position est désormais clairement affirmée. D'autant qu'à l'échec de la départementalisation, le facteur interne, s'ajoute le facteur externe. A cette époque, dans la région caribéenne, les pays sous domination anglaise ou hollandaise sont pourvus d'un Exécutif local. Tel est le cas de la Jamaïque, de Trinidad, du Surinam. En Afrique du Nord, la Tunisie et le Maroc accèdent à l'indépendance. Quant aux pays d'Afrique Noire Francophone, la loi-cadre du 23 juin1956 les dote d'institutions nouvelles. Ils bénéficient d'un embryon d'Exécutif territorial qui permet aux autochtones de participer aux responsabilités de l'action quotidienne dans les domaines qui leur sont transférés.

La décision du PPM de réclamer un changement de statut - Césaire étant celui qui définit la ligne - trouve son fondement non pas dans la condamnation de l'assimilation mais dans son application jugée inéquitable. Il faut ajouter, et le point ne nous parait pas négligeable, que la petite bourgeoisie martiniquaise, éduquée, aux revenus souvent confortables supporte mal que contrairement à la situation africaine ou à celle de ses voisines caribéennes elle soit exclue du Pouvoir politique ou de son apparence.

L'embarras est patent quand il s'agit de proposer un statut nouveau pour remplacer la départementalisation. Dans un premier temps, au moment de sa fondation, le PPM se déclare partisan de «La transformation de la Martinique en région dans le cadre d'une Union Française fédérée ${ }^{13}$. Il considère que même hors le cadre fédéral, la République italienne «une et indivisible » est «diversifiée et décentralisée et cela grâce à l'introduction dans la Constitution [italienne] de la notion de «région ». Tel est le principe. $\mathrm{Au}$ plan organisationnel Aimé Césaire est attiré par un modèle fédéral

\footnotetext{
${ }^{11}$ A. Césaire. «Préface » in D. Guérin. Les Antilles décolonisées. Paris : Présence Africaine, 1956, p.13.

${ }^{12}$ Le Monde Hebdo. 6-11 avril 1961.

${ }^{13}$ A. Césaire. Euvres complètes. Tome III. Fort-de-France : Ed. Désormeaux, 1976, p. 477 et suiv.
} 
particulier, celui de l'URSS. Il distinguait les républiques fédérées, les républiques autonomes, les régions autonomes et les districts autonomes. De ce point de vue, la Martinique ne constituerait pas une «République fédérée » mais une «Région ». Dans la même période, il est parfois fait appel à l'autogestion sans que son contenu soit jamais clairement précisé. La souveraineté n'est pas à l'ordre du jour.

La radicalisation apparaît avec la visite effectuée à la Martinique par le Général de Gaulle en mars 1964. Césaire, toujours lui, parle de «la nécessaire refonte des institutions locales (...) pour qu'elles soient plus respectueuses de notre particularisme évident faisant davantage place à la responsabilité locale». Le Général rétorque avec brutalité qu' «entre l'Europe et l'Amérique, il n'y a que des poussières et on ne construit pas des Etats sur des poussières ». Grande déception. La gauche antillaise ou ce qu'il est convenu d'appeler ainsi s'attendait à une autre réaction de la part de 1 «Homme du 18 juin» d'autant que ce dernier, avant le réferendum de 1958, avait adressé un «message » aux départements d'outre-mer, La Réunion ne semblait pas concernée, dans lequel il indiquait que la République entendait maintenir aux Antillais et aux Guyanais leurs « franchises » et que les élus de ces pays participeront à l'adaptation des lois aux nécessités locales ${ }^{14}$.

\section{L'AUTONOMIE OU L'AUBERGE ESPAGNOLE}

Après la «sortie» du Général de Gaulle, le discours PPM va changer de tonalité et l'autonomie sera réclamée «...Tout ce qui va dans le sens du régionalisme en France joue pour la Martinique dans le sens de l'autonomie $»^{15}$. Telle est la position adoptée en août 1967. Le statut revendiqué tire donc du côté du régionalisme sinon de la régionalisation. Il n'est, donc, pas question de souveraineté.

En mars 1968, soit quelques mois après la prise de position dont il vient d'être fait état, l'existence de la nation est affirmée avec netteté : «A l'heure actuelle, il faut sans ambages appeler nations, les groupes humains que

\footnotetext{
${ }^{14}$ Le Monde. 19 septembre 1958.

${ }^{15}$ « Discours de clôture du III ${ }^{\mathrm{e}}$ Congrès du PPM ». 13 août 1967 in Euvres Complètes, op. cit. p. 507.
} 
constituent chacune pour sa part la Martinique et la Guadeloupe ${ }^{16}$. Il en découle cette importante conséquence : «Etant nation, la Martinique a comme toutes les nations le droit de disposer librement d'elle-même $»^{17}$. Le PPM, indique son président, a choisi l'autonomie, c'est-à-dire «ratification de l'existence de la Martinique en tant que peuple et faculté pour les Martiniquais d'organiser leur propre vie collective et de s'administrer eux-mêmes ${ }^{18}$. Cette conception a été perçue comme se situant dans la problématique de la souveraineté. La Convention pour l'autonomie tenue en août 1971 et réunissant diverses formations politiques des DOM renforce ce sentiment. En effet, son document final affirme : «Les peuples des quatre territoires de $\mathrm{La}$ Réunion, de la Guyane, de la Guadeloupe, de la Martinique constituent (...) des entités nationales. [Celles-ci] doivent être constituées dans le cadre juridique d'un Etat autonome ${ }^{19}$. Il y a là de manière implicite une référence à l'ancien Titre XII de la Constitution de 1958 instituant la Communauté. Ce rapprochement n'est pas sans intérêt lorsque l'on se souvient que tous les Etats membres de la Communauté se sont rapidement prévalus de l'article 86 de la Constitution prévoyant: «Un Etat membre de la Communauté peut devenir indépendant ». De plus, la conviction que l'Etat, c'est-à-dire la souveraineté, c'est-à-dire l'indépendance - mais il est prématuré d'en parler - est, à terme, la «couverture juridique » de l'autonomie peut s'appuyer sur le mot d'ordre adopté par le Congrès de 1980 : «Autonomie pour la nation martiniquaise, étape de l'histoire du peuple martiniquais en lutte depuis trois siècles pour son émancipation définitive ». Il est vrai que l'on peut disserter longuement sur la notion d' «émancipation définitive »... Ne perdons pas de vue que jusqu'à une époque récente la grande majorité des juristes de Droit public considérait que l'Etat était la traduction juridique de la nation. Ainsi Carré de Malberg estimait : «en ce sens juridique, la nation n'est plus seulement un des éléments constitutifs de l'Etat, mais elle est, par excellence l'élément constitutif de l'Etat, en tant qu'elle s'identifie avec lui $»^{20}$. L'analyse est aujourd'hui plus nuancée mais, à l'époque, c'est elle qui prévalait.

\footnotetext{
${ }^{16}$ « Allocution pour le $\mathrm{X}^{\mathrm{e}}$ anniversaire de la fondation du PPM » 22 mars 1968 in Euvres Complètes, op. cit. . p519.

${ }^{17}$ Ibidem, p. 520.

${ }^{18}$ Ibidem, p.521.

${ }^{19}$ Convention pour l'autonomie. Morne-Rouge. 16, 17,18 août 1971. Brochure éditée par le Parti Communiste Martiniquais.

${ }^{20}$ R. Carré De Malberg. Contribution à la théorie générale de l'Etat. Paris : Rééd. CNRS, 1985, tome I, p.16.
} 
Procédant à un intéressant rapprochement avec Porto Rico, Justin Daniel estime que le «nationalisme culturel » s'est substitué au «nationalisme politique $»^{21}$. On peut également penser aux thèses qui avaient cours en France, il y a une trentaine d'années sur le «nationalitarisme $»^{22}$.

La lisibilité de la position du PPM est rendue difficile par le fait que dans la même période, il arrive qu'il assigne comme objectifs à l'autonomie la réalisation de progrès dans le domaine économique et social de même que le respect de l'identité culturelle dans le cadre français. L'autonomie doit, alors, permettre d'obtenir ce qui était escompté de la départementalisation et qui n'a pas été obtenu. De fait, l'autonomie est aujourd'hui un mot polysémique. Il désigne un statut de transition vers la souveraineté, ce qui implique, à terme, la séparation par rapport à l'Etat dominant mais aussi un aménagement de l'organisation de l'Etat dominant dont les structures ne sont pas mises en cause.

La campagne du PPM à l'occasion des élections législatives de mars 1978 illustre ce cas de figure: «L'autonomie n'est ni un passéisme ni un misérabilisme. C'est un plus et ce n'est pas un moins. Ce qui est acquis restera acquis. Mais ce qui manque sera ajouté ${ }^{23}$. C'est disions-nous l' «autonomiestade suprême de l'assimilation $»^{24}$, une assimilation modernisée, aménagée, raffinée mais ne gommant pas le lien de dépendance fort avec la France. La formule est peut-être excessive. La position du PPM est, à vrai dire, délicate. Il

Observons qu'aujourd'hui, en Espagne, s'agissant de la Catalogne et du Pays basque est affirmée la compatibilité de la nation et de l'autonomie. Le PPM soutenait ce point de vue, il y a maintenant plus de trente ans : "On ne peut que constater que la communauté humaine vivant à la Martinique forme, indiscutablement, un peuple particulier par rapport à la communauté humaine vivant en France. La nation ne veut pas dire tel ou tel régime politique. $\mathrm{La}$ nation correspond à un peuple. Le régime politique correspond à un statut ». Le Progressiste. 20 au 24 juillet 1974. No 537.

21 «Les élus face à la réforme institutionnelle et à l'acte II de la décentralisation : la difficile conciliation d'aspirations contradictoires » in Thierry Michalon (dir.) Entre assimilation et émancipation. L'outre-mer français dans l'impasse ?, Rennes, Les Perséides, 2006, pp. 113-132. ${ }^{22}$ Voir, par exemple, M. Oriol. «Identité produite, identité instituée, identité exprimée : confusions des théories de l'identité nationale et culturelle ", Cahiers internationaux de sociologie, vol. LXVI, 1979. Les notions de nation et d'autonomie ne sont pas incompatibles. Enfin, le débat qui a cours en France sur l'identité plurielle donne d'une certaine manière un nouvel éclairage à la question.

${ }^{23}$ Le Progressiste, 25 février 1978.

${ }^{24}$ Notre thèse : Compère Lapin et Compère Mulet. Métissage et comportements socio-politiques à la Martinique. Paris IX - Dauphine, 1988. p. 335. 
affirme des positions de principe dans les conditions que nous avons évoquées mais dans le même temps il doit tenir compte de la réalité politique. Du fait d'un découpage électoral inique, il ne contrôle que Fort-de-France, qui, à certaines périodes fait figure de citadelle assiégée, le gouvernement lui est franchement hostile et, alors même, nous l'avons vu, qu'il se garde bien de parler d'indépendance, il est accusé d'en nourrir le projet. Malgré l'admiration et la confiance que beaucoup de Martiniquais éprouvent pour Aimé Césaire, ceux-ci sont rétifs à tout changement politique. Pour deux raisons. La première est qu'il leur est donné en exemple la situation économique et sociale très difficile des pays de la Caraïbe, l'échec des indépendances africaines, tel est du moins le discours qui est tenu. La seconde est que la décennie soixante dix marque l'entrée effective de la Martinique dans la société de consommation. Nombreux sont les Martiniquais qui, à cette époque, reconnaissent que l'économie est dans une situation très alarmante, caractérisée par le paradoxe de la non production et de la surconsommation selon l'heureuse formule de Michel Louis ${ }^{25}$. Ils se plaignent aussi que leur personnalité propre est, pour l'essentiel, niée. Mais ils sont fortement attachés aux acquis sociaux et à leur niveau de vie. La marge du PPM est donc très limitée. Il faut aussi tenir compte de la trajectoire particulière du peuple martiniquais et du caractère atypique de cette société. Les responsables du PPM appartenant dans leur grande majorité à ce que l'on appelle généralement la bourgeoisie mais qui n'est, en fait, qu'une bourgeoisie de représentation, à vrai dire la frange supérieure de la petite bourgeoisie aspirent à l'exercice du pouvoir ${ }^{26}$. Avec l'adhésion au Parti, en 1978 de militants venus du Parti Socialiste Martiniquais

\footnotetext{
${ }^{25}$ Michel Louis. L'échange idéal. Essai sur une problématique de la consommation en Martinique. Thèse de doctorat d'Etat. Paris IX — Dauphine. Juin 1981, dact.

${ }^{26}$ A la Martinique, le bourgeois n'est pas celui qui possède un moyen de production. C'est un individu qui a, certes, un niveau élevé de revenus mais qu'il tire non du secteur primaire ou du secteur secondaire mais du tertiaire. On est en présence d'une bourgeoisie de représentation et non pas de fonction. L'élément décisif est la place occupée dans la société de consommation. Le bourgeois est celui qui peut dépenser et paraître. Si l'on considère que l'analyse marxiste n'est pas complètement obsolète, on peut admettre que la notion de quasi-bourgeoisie doit être substituée à celle de bourgeoisie. Michel Louis (op. cit.) souligne le rôle de la dépense comme signe d'assomption. L' "effet de monstration», dit-il. De son côté, Francis Affergan (Anthropologie à la Martinique, Paris, Presses de la Fondation Nationale des Sciences Politiques, 1983, p. 49) fait ressortir que la petite bourgeoisie - il vise, en fait, la « bourgeoisie martiniquaise » — «déborde largement d'un lieu d'ancrage socio-économique, stricto sensu, pour épouser plutôt un contour symbolique ». C'est, évidemment, une analyse qui emprunte à la pensée de Pierre Bourdieu.
} 
qui s'est auto-dissous, le PPM réaffirme sa position nationaliste et se déclare partisan du socialisme démocratique. Mais, pour ces responsables, le pouvoir revendiqué ne remet pas en cause leur niveau de vie. Ils ne l'envisagent pas plus que ne l'envisage le peuple martiniquais. Ils ne sont pas préparés à la rupture, fût-elle limitée ${ }^{27}$. Les conséquences, au plan martiniquais, de l'arrivée de la gauche au pouvoir en France le montrent bien.

\section{LA GESTION DU MORATOIRE OU LE RENONCEMENT AU NATIONALISME}

François Mitterrand, élu président de la République, Aimé Césaire et le PPM considèrent que la donne a changé. Désormais, le Pouvoir sera plus attentif à la cause martiniquaise mais ils intègrent aussi le fait qu'à la Martinique Valéry Giscard d'Estaing l'a emporté nettement. Le 29 mai 1981, dans un discours solennel tenu à la Mairie de Fort-de-France, le président du PPM décrète un «moratoire » sur la question du statut. La décision est bien accueillie. Des élections régionales ont lieu le 21 février $1983^{28}$. La liste conduite par Aimé Césaire l'emporte et celui-ci est élu président de la région. Camille Darsières, premier vice-président et secrétaire général du PPM assure l'effectivité de la charge ${ }^{29}$. Il inscrit donc son action dans la logique du moratoire. Peut-être peut on rappeler que Le Petit Larousse Illustré (Ed. 2005) définit ainsi le moratoire: «suspension

\footnotetext{
${ }^{27}$ Dans le cadre de cet article, nous ne pouvons développer ce point et nous renvoyons à notre thèse. En particulier aux pages 231 et suivantes où reprenant une idée de Jean Price-Mars (Ainsi parla l'Oncle. Ed. Lemeac, Montréal, 1973, p.44), nous suggérons que le Martiniquais serait atteint de «bovarysme collectif », c'est-à-dire que la société martiniquaise se concevrait autre qu'elle n'est. Et que le Martiniquais est peut-être justiciable de l'analyse de Kojeve (A. Kojeve. Introduction à la lecture de Hegel. NRF - Gallimard, Paris, 1976).

28 Sur la base des lois de décentralisation du 2 mars et du 31 décembre 1982 portant organisation des régions de Guadeloupe, de Guyane, de Martinique et de la Réunion et définissant le compétences de la région. Elles permettent l'organisation et le déroulement exclusivement au sein de Départements d'Outre-Mer des premières élections régionales au suffrage universel direct dès le 21 février 1983. A propos des élections régionales, il faut citer Lucie Jean-Louis : Etude comparative des scrutins régionaux de la Guadeloupe et de la Martinique: profils sociologiques des candidats et des élus des assemblées régionales de 1986 à 2004. Mémoire pour le DEA «Relations internationales et politique comparée », Université des Antilles et de la Guyane, Septembre 2004.

${ }^{29}$ Il deviendra effectivement président en 1988 soit deux ans après le renouvellement de 1986. Il perdra la Présidence lors du renouvellement de l'Assemblée régionale en 1992.
} 
volontaire d'une action; délai que l'on s'accorde avant de poursuivre une activité dans un domaine donné ». Il s'agit donc d'une halte, d'une pause. D'ailleurs Le Progressiste ${ }^{30}$ (15 juillet 1981) ne laisse pas place au doute : «Soyons clairs et soyons nets : la Déclaration du Comité National du Parti rendue publique le 24 mai n'a jamais proclamé la fin du mot d'ordre d'autonomie. Personne n'a jamais requis la convocation d'un Congrès qui exige que l'on efface de nos tablettes le mot d'ordre arrêté par le Congrès de 1980. Cela signifie sans ambiguïté aucune que le PPM a un combat fondamental résumé par son mot d'ordre: «Autonomie pour la nation martiniquaise, étape de l'histoire du peuple martiniquais en lutte depuis trois siècles pour son émancipation définitive ». Et l'auteur de l'article insiste : «L'autonomie n'est pas renvoyée aux calendes ». La remarque n'est évidemment pas gratuite. C'est que, dès le début, certains militants PPM s'interrogent sur la nature du moratoire et, le temps passant, la pause parait bien longue. La question est posée de savoir si le moratoire ne masque pas un changement de cap. Et si, contrairement à la déclaration de juillet 1981, l'autonomie n'est pas «renvoyée aux calendes » et le nationalisme enterré ou à tout le moins réduit à sa dimension culturelle. Plus, précisément, l'autonomie n'est-elle pas un autre nom de la décentralisation de droit commun? L'interrogation se justifie d'autant plus que la décentralisation, acte II répond à bon nombre des revendications portant sur la domiciliation de certaines compétences et que par ailleurs sur le plan de la reconnaissance de l'identité culturelle de réels progrès ont été accomplis.

Il faut observer qu'au sein du PPM, le moratoire a eu pour conséquence le départ des militants «indépendantistes» et l'arrivée de personnalités venant d'horizons divers. Des dirigeants du Groupe Révolution Socialiste (GRS) qui avaient apparemment abjuré leur foi indépendantiste, des membres de la société civile qui jusque là ne s'étaient manifestés que comme des «démocrates ». Et même un ancien secrétaire fédéral du RPR. Ce PPM «relooké » conquiert outre (pour un temps) la présidence du Conseil régional, celle (plus durablement) du Conseil général et plusieurs cantons et communes.

Force est aussi de constater qu'au Congrès des élus départementaux et régionaux tenu les 20, 21 et 23 février 2002, le PPM «ne prend pas part

\footnotetext{
${ }^{30}$ Organe officiel du PPM.
} 
au vote » de la motion «tendant à l'existence d'une nation martiniquaise $»^{31}$. Et il soutient bien mollement le OUI - à l'exception notable de Claude Lise, sénateur et président du Conseil général - lors de la consultation populaire du 7 décembre $2003^{32}$. Pour faire image, on peut dire que la position du Parti fait penser à un cheval de concours qui, lancé, regimbe au dernier moment devant l'obstacle.

A quoi cela tient-il ? A la peur de l'avenir. A la volonté de marcher au même pas que le peuple. Pour une part. Mais plus fondamentalement, pensons-nous, à notre histoire particulière, à notre présent singulier (le paradoxe de la non production et de la surconsommation). En d'autres termes, l'assimilation s'est profondément et insidieusement ancrée chez beaucoup de Martiniquais, y compris les plus déterminés à lutter contre elle. Affaire de bulbe rachidien ${ }^{33}$ !

Pour être complet, il faut rappeler que le maire de Fort-de-France et nouveau président du PPM, Serge Letchimy, a écrit un Discours sur l'Autonomie $^{34}$ dans lequel il s'efforce de préciser la notion. Et que surtout, le congrès de juin et d'octobre 2005, dit Congrès de la Clarification ${ }^{35}$, préconise un nouveau mot d'ordre : «Autonomie pour la Nation Martiniquaise, étape de l'Emancipation de l'Homme et du Peuple Martiniquais ». Et, pour ne pas sortir complètement du clivage traditionnel droite/gauche, il se définit comme étant à «idéal socialiste». Comment sera-t-il appliqué ? Sur ces bases, on peut penser que la revendication, au plan institutionnel, devrait être de demander de bénéficier des dispositions de l'article 74 de la Constitution qui permettrait, entre autres, d'obtenir - mais ce n'est pas automatique - la reconnaissance de signes distinctifs de l'identité.

Les Indépendantistes en général et le MIM en particulier ont une position plus claire sur la nation. Mais l'analyse peut révéler des surprises.

\footnotetext{
${ }^{31}$ Il faut noter qu'un élu PPM, Renaud de Grandmaison a voté la motion.

${ }^{32}$ A l'occasion de ses 99 ans, Pierre Aliker, compagnon de tout temps d'Aimé Césaire et membre très influent du PPM a reconnu avoir voté NON.

${ }^{33}$ Pour reprendre une formule qu' affectionne un de nos amis.

${ }^{34}$ Martinique, Ibis Rouge Editions, 2002.

${ }^{35}$ Un certain nombre de militants regroupés autour de Claude Lise ont refusé de participer au Congrès au motif principal qu'il fallait un Congrès de la Refondation. Certes, «clarification » n'est pas «refondation». Mais, développées par les tenants de l'une ou l'autre notion, les différences paraissent bien ténues. Serait-on encore dans le brouillage ?
} 


\section{B - LE MIM OU LE NATIONALISME INCANTATOIRE}

En un peu plus de trente ans ${ }^{36}$ et si l'on prend comme point de repère la représentation au Conseil régional, le Mouvement Indépendantiste Martiniquais est devenu la première force politique du pays. Il revendique l'Indépendance alors que les Martiniquais, dans leur grande majorité, y sont résolument hostiles.

Cependant, sa trajectoire est moins rectiligne qu'il n'y parait. La rupture proclamée se traduit dans les faits par une politique pragmatique (1) qui cherche à concilier l'affirmation identitaire et la gestion du système (2).

\section{LA POLITIQUE DES PETITS PAS}

Revendiquant l'indépendance, le MIM est, selon la grille martiniquaise, classé à l'extrême gauche. Mais si on lui applique les critères traditionnels, on peut s'interroger sur la pertinence de ce classement. Il est vrai que la littérature du MIM n'est pas très abondante et que, par conséquent, il y a peu de textes définissant la ligne de l'organisation. A notre connaissance, les références au socialisme, par exemple, sont inexistantes ou à tout le moins rares et vagues surtout depuis que Marc Pulvar, l' «idéologue» de la formation n'y milite plus. L'organisation ne semble guère concernée par l'alter-mondialisme, par exemple. De plus, la pratique et les discours du leader charismatique du MIM, Alfred Marie-Jeanne ne vont guère dans le sens du progressisme. Il ne fait pas mystère de son inclination pour les méthodes

\footnotetext{
${ }^{36}$ En 1971, Alfred Marie-Jeanne est élu en même temps que trois autres « démocrates », maire d'une commune du Sud de la Martinique, Rivière-Pilote. Les autres sont élus à Ducos, RivièreSalée et au François. C'est un événement. Les maires sortants, battus, sont classés à droite, donc partisans du statu quo départemental. A ce moment là, sur la question du statut, le maire de Rivière Pilote n'exprime pas de position particulière. Il va rapidement évoluer. En 1973, il est candidat aux élections législatives dans la circonscription Sud de la Martinique. Il fédère autour de lui un petit nombre de groupuscules indépendantistes, le plus souvent maoïstes. N'oublions pas que mai 1968 n'est pas loin. Il a pour suppléant, un militant PPM, le Dr Désiré qui sera par la suite, Sénateur de la Martinique. En l'absence de Césaire, des responsables PPM conviennent que Césaire dans le Centre et Marie-Jeanne, dans le Sud feront campagne sur le thème de l'autodétermination avec pour mot d'ordre «La parole au peuple». Déroute pour Marie-Jeanne. Césaire est réélu de justesse. Et le PPM en revient à sa position classique. Le mot d'ordre «La parole au peuple » devient le nom de l'organisation que le maire de Rivière-Pilote crée avec ses nouveaux amis. En 1978, il devient le MIM (Mouvement pour l'Indépendance de la Martinique) qui se transforme par la suite en Mouvement Indépendantiste Martiniquais.
} 
traditionnelles d'éducation. Il ne dissimule pas davantage sa réticence vis-à-vis des intellectuels, les «donneurs de leçons » et, en particulier de ceux qu'il appelle les «marqueurs de parole»: «Il y a une différence entre écrire et se battre pour cela. (...) Comment allons-nous augmenter la conscience du peuple si nous sommes des indépendantistes mais en parole, en parole seulement ? ${ }^{37}$. Bref, la posture est plutôt conservatrice.

Cependant, il était permis de penser que l'alliance avec le Conseil National des Comités Populaires (CNCP), nouée peu avant les régionales de 2004, marquerait sans contestation possible le positionnement du MIM à l'extrême gauche, son allié étant à cet égard d'une clarté absolue. Une telle conclusion est peut-être hâtive. Quelle est la nature de l'alliance ? Pour l'heure, on ne voit pas de trace de l'influence du CNCP sur les positions traditionnelles du MIM. Le rapport de forces est trop inégal ${ }^{38}$. Les militants du CNCP sont, comme à leur habitude, présents sur le terrain et ceux d'entre eux qui exercent des responsabilités au Conseil régional le font avec un très grand sérieux. On ne peut en dire plus...

Ceci posé, il faut reconnaître que le MIM et son chef de file font preuve de réalisme. Dans un premier temps, ils ont en quelque sorte marqué leur territoire. Retranchés à Rivière Pilote, ils refusaient tout dialogue avec le Pouvoir, prônant l'abstention aux «élections françaises». En clair, cela signifiait le refus de voter aux élections législatives, présidentielles et aux référendums et l'incitation à participer aux scrutins intéressant directement les Martiniquais, c'est-à-dire les régionales, les cantonales et les municipales. La position a évolué. Le MIM est désormais présent aux élections législatives et Alfred Marie-Jeanne est, rappelons-le, député de la circonscription Sud de la Martinique. Pendant les campagnes électorales, il n'omet pas de souligner qu'il milite pour l'indépendance mais que tel n'est l'enjeu ni des législatives ni des régionales. L'évolution se marque aussi par le fait que le dialogue avec le gouvernement est aujourd'hui engagé, du moins tel était le cas tout le temps que Mme Girardin était Ministre de l'outre-mer.

\footnotetext{
${ }^{37}$ «Conférence de rentrée du MIM ». 28 septembre 2005 in Antilla ${ }^{\circ}$ 1163, 5 octobre 2005.

${ }^{38}$ Sur 28 élus de la liste «Groupe des Patriotes MIM-CNCP», il y a trois représentants du $\mathrm{CNCP}$ dont, il est vrai, le second vice-président de l'assemblée, Marie-Hélène Leotin et le président de la commission «Culture », Edmond Mondésir. Le troisième élu issu du CNCP est Josette Massolin.
} 
Indépendantiste, nationaliste. Assurément. Observons toutefois que les militants du MIM n'emploient d'habitude pour se désigner aucun de ces deux qualificatifs. Ils se disent «patriotes ». Ne sommes-nous là que dans la nuance sans réelle importance ou, au contraire, ne s'agit-il pas de la volonté de rassurer en utilisant un terme moins connoté politiquement ?

En toute hypothèse, le MIM n'effraie plus aujourd'hui, en tout cas beaucoup moins que par le passé, en grande partie parce que l'électeur a appris à distinguer la nature des enjeux et qu'il ne respecte plus nécessairement les consignes du maire qui, pendant longtemps, a été, dans une certaine mesure, le substitut du maître opérant à l'habitation.

Ainsi, aux élections législatives de juin 2002, dans un contexte de changement institutionnel annoncé, le chef de file du MIM n'a conservé son siège que d'extrême justesse. En effet, il ne l'a emporté que de 750 voix alors qu'il avait distancé le même adversaire aux élections précédentes de 12000 voix et aurait vraisemblablement été battu dès le $1^{\text {er }}$ tour si deux candidats de la gauche traditionnelle, selon la terminologie en usage, ne s'étaient présentés. Lors de la consultation populaire du 7 décembre 2003, il s'implique clairement en faveur du vote pour le OUI, le NON gagne ${ }^{39}$. Aux élections régionales des 21 et 28 mars 2004, la liste qu'il conduit est littéralement plébiscitée.

Comment expliquer ce paradoxe? Nombre de Martiniquais redoutaient que la nouvelle collectivité, malgré les verrous ${ }^{40}$ mis en place, ne débouche sur l'aventure, sinon l'indépendance, en tout cas un statut qui, serait-ce à terme et même très partiellement, remettrait en cause l'appartenance à la République française et à l'Union Européenne. Nous ne sommes plus là dans la pulsion mimétique mais dans le concret, le palpable, la sauvegarde du niveau de vie. De plus il arrivait que certains confient qu'ils redoutaient une Assemblée unique présidée par Marie-Jeanne qui, d'après eux, n'est point l'ami des libertés.

\footnotetext{
${ }^{39}$ La question était la suivante : «Approuvez-vous le projet de création en Martinique d'une collectivité territoriale demeurant régie par l'article 73 de la Constitution, et donc par le principe de l'identité législative avec possibilité d'adaptations, et se substituant au département et à la région dans les conditions prévues par cet article ?».

${ }^{40}$ Le mot est d'André Oraison, «Quelques réflexions générales sur l'article 73 de la Constitution de la $\mathrm{V}^{\mathrm{e}}$ République, corrigé et complété par la loi constitutionnelle du 28 mars $2003 »$. Revue juridique et politique, 2003, N 2.
} 
Marie-Jeanne et le MIM ont compris depuis longtemps que l'indépendance est rejetée par la très grande majorité des Martiniquais. Ils continuent, pourtant, à s'y référer. De manière quasi rituelle. Pour se persuader, peut-être, qu'ils sont toujours en accord avec leur idéal de jeunesse. Telle n'est pas la question. C'est la situation politique, économique, sociale, l'environnement international qui fait que l'indépendance n'est pas envisageable à terme prévisible. Mais des avancées modestes eu égard à l'objectif proclamé sont possibles.

Ainsi, Alfred Marie-Jeanne est à l'origine d'une initiative importante et innovante. En effet, le 18 janvier 2000, le Conseil régional a décidé de bâtir un projet global pour la Martinique et a arrêté de mettre en place à cette fin une commission ad hoc, tous les groupes étant représentés selon la règle proportionnelle. La commission s'est entourée d'un secrétariat permanent composé d'experts indépendants ${ }^{41}$ et a décidé que le Forum final serait précédé d'un travail en ateliers, lesquels comprendraient des responsables politiques et des opérateurs du monde socioprofessionnel ${ }^{42}$.

L'intérêt a été réel et le 6 octobre 2000 les experts ont présenté au public le «Projet Martinique - Projet de développement économique, social et culturel ». Les différents ateliers ont émis des propositions qui, dans l'ensemble, ne sont pas d'une grande originalité mais ont le mérite d'exister. En particulier, les travaux de l'atelier «Institutions », amendés par la commission ad hoc et, semble-il, par la suite par le président lui-même ont servi de base de travail au Congrès des élus départementaux et régionaux. Les résolutions de ce Congrès ont ouvert la voie à la consultation populaire. Elles allaient sur des points essentiels bien au-delà de la réforme proposée par le gouvernement.

Dès lors, on était en droit de considérer que la cause était entendue et que les Martiniquais, dans leur majorité, se prononceraient en faveur de la réforme qui leur était proposée. Tel n'a pas été le cas mais, rassurés sur leur maintien dans la République et leur appartenance à l'Union Européenne, ils entendent que soit reconnue leur identité propre.

${ }^{41}$ Antillais. Le point mérite d'être souligné.

${ }^{42}$ Sept ateliers ont été mis en place : Institutions, Culture, Social, Economie et Développement Durable, Coopération Régionale et Internationale, Education-Formation, Environnement et Aménagement. Les ateliers devaient être animés par les experts et il était prévu que chacun d'eux se réunirait trois fois. Par ailleurs, la démarche reposait sur « la mobilisation des citoyens par tous les moyens en particulier par la tenue de séances de travail publiques ». 


\section{L'IDENTITE SECURISEE ET LA GESTION DE BON PERE DE FAMILLE}

Marie-Jeanne est, aux yeux de beaucoup, le meilleur porte-parole de la volonté identitaire. Les Martiniquais veulent concilier nationalité française et identité caribéenne. Ils ont choisi «l'identité sécurisée ${ }^{43}$ et ils souhaitent aussi que la collectivité régionale soit gérée sans risques.

Au plan identitaire - nous ne visons pas l'exhaustivité - une attention particulière est portée à la mise en valeur et à la conservation du patrimoine sous toutes ses formes. Ainsi, il existe un musée régional d'histoire et d'ethnographie mis en place, il est vrai, sous la présidence Darsières. Un effort particulier est porté au devoir de mémoire, à la restitution de l'histoire. De ce point de vue, il arrive que le MIM et ses alliés n'échappent pas totalement à la tentation d'instrumentaliser l'histoire. Ainsi, Alfred Marie-Jeanne a déclaré : «Le 22 mai 1848 marque la date de la fondation de la nation martiniquaise ». Sans entrer dans le fond de la question, on peut se demander si la création d'une nation a date certaine ${ }^{44}$. Par ailleurs, il y a en préparation un projet concernant l'éducation, une forte implication dans les manifestations culturelles et sportives concernant la Caraïbe et, de manière plus générale, une politique de coopération régionale très active.

Le MIM conquiert assez rapidement l'électorat du Conseil régional. Lors des premières élections $(1983,1986)$ la liste qu'il présente n'obtient aucun siège ${ }^{45}$ mais en 1990 - l'élection de 1986 ayant été annulée - il recueille 7 sièges. En 1992, il passe à 9 sièges, à égalité avec le PPM qui préside l'Assemblée depuis 1983. En 1998, il en est à 13 sièges et Alfred Marie-Jeanne est élu président du Conseil régional ${ }^{46}$. En 2004, avec son allié le $\mathrm{CNCP}$, il a 28 sièges représentant les deux tiers du Conseil.

\footnotetext{
${ }^{43}$ La formule est de Julien Mérion. Conférence prononcée le 18 juillet 2004 in «Le Cénacle ». $34^{\mathrm{e}}$ Festival Culturel de Fort-de-France.

${ }^{44}$ Discours à Rivière-Pilote, le 22 mai 1986.

${ }^{45}$ Le Conseil régional comprend 41 élus.

${ }^{46}$ La droite - La liste de l'Union - est majoritaire en sièges (elle en a 14) mais Marie-Jeanne bénéficie pour l'élection à la Présidence de voix d'élus de gauche. Il s'ensuit une participation très active de Pierre Petit, tête de la liste de l'Union, à la direction de l'Assemblée. On ne pourrait, cependant, parler de co-gestion. Marie-Jeanne y gagne une réputation d'homme d'ouverture qu'il n'avait pas véritablement jusque là.
} 
Depuis ses débuts à Rivière Pilote alors qu'il ne pouvait compter ni sur l'Etat ni sur le Conseil général, l'actuel président du Conseil régional s'est acquis une réputation de bon gestionnaire. Il s'en explique clairement : «On a trop souvent dit que les patriotes, les indépendantistes étaient incapables de gérer, nous leur avons démontré le contraire. La Martinique est au bord de la faillite et beaucoup de communes devraient se trouver devant la Cour des Comptes. Oui, et puis deux fois oui et encore mille fois oui : la bonne gestion fait partie de la tactique et de la stratégie politique des patriotes, car comment donner au peuple confiance, si vous ne savez pas gérer? ${ }^{47}$. Ses adversaires rétorquent qu'il est «assis sur un matelas d'argent» qu'il utilise à mauvais escient, ou plutôt qu'il n'utilise pas, n'ayant pas compris que les fonds d'une importante collectivité publique ne se gèrent pas comme le panier de la ménagère. Bref, sa gestion serait archaïque. Jean Crusol, ancien président de la Fédération Socialiste et Professeur d'Economie le dit sans détour: «Le taux de croissance de l'économie qui était de 3,6\% en 1998 est tombé à $0,7 \%$ en $2000(\ldots) \mathrm{La}$ dépense et l'investissement de la région ont diminué et les sommes stérilisées à la région ont atteint des sommets astronomiques ${ }^{48}$. Reste que les Martiniquais sont rassurés par sa manière de faire.

Au-delà des discours, des mots d'ordre, du poids du passé, des oppositions, à vrai dire, naturelles entre les hommes, il nous semble qu'il y a une réelle convergence entre les positions du PPM et celles du MIM. L'une et l'autre formation politique se réclament avec force de l'identité martiniquaise de même qu'elles souhaitent rester dans le cadre de la République et de l'Union Européenne. L'idée d'une nouvelle réunion du Congrès des élus départementaux et régionaux afin de réexaminer la question institutionnelle a été lancée par le président du Conseil régional. Il est vrai qu'elle n'a pour l'instant pas trouvé d'écho auprès du Conseil général. Mais il s'agit d'institutions. Les organisations politiques, elles, n'excluent pas d'obtenir dans un terme qui ne serait pas trop éloigné une nouvelle consultation et, dans ce cas, il n'est pas inenvisageable que la demande consiste à solliciter le statut prévu par l'article 74 de la Constitution. Politique-fiction ? Pas sûr. En deux ans, il n'est pas impossible que les risques liés à l'abandon du statut

\footnotetext{
47 «Conférence de rentrée du MIM », 28 septembre 2005, in Antilla., n 1163, 5 octobre 2005.

48 Interview à France Antilles. 11 mars 2004. Par ailleurs, dans un document, non daté, dact., intitulé : Notes sur le compte administratif 2004 de la région, Jean Crusol fait ressortir que les dépenses qui assurent le développement économique diminuent en fait de 3\% par rapport à 2002.
} 
départemental aient été reconsidérés et qu'ils apparaissent moins dommageables aujourd'hui. Parallèlement, il n'est pas inimaginable que les citoyens soient séduits par un statut garantissant les acquis et offrant à la fois une plus grande marge de manœuvre dans la gestion des affaires et permettant une réelle affirmation de l'identité.

La même convergence - que nous posons en hypothèse - existe-t-elle avec la droite?

\section{DE L'ASSIMILATIONNISME A LA REVENDICATION IDENTITAIRE}

La satisfaction du désir de reconnaissance a pris la forme de la pulsion mimétique. Détour obligé. La trajectoire particulière de la Martinique - société atypique, soutenons-nous - a eu pour conséquence que l'assimilation ne se limite pas à l'intégration-dont on voit bien les limites - elle se traduit par la volonté de fusion. Les hommes de couleur libres, disons par commodité les mulâtres, veulent se voir reconnaître comme hommes et, corrélativement, faire oublier leur part nègre. Ils mettent un point d'honneur à apparaître, aux yeux des blancs, comme des civilisés, des gens raisonnables. D'ailleurs, s'ils réclament des droits, ils insistent aussi pour payer "l'impôt du sang »" Dans le même ordre d'idées, on peut se demander si l'acharnement à «payer l'impôt du sang », la volonté de mêler sur le champ de bataille son sang à celui du Français de France ne répond pas au désir d'affirmer, plus encore que l'égalité, la consanguinité. L' «ordre du jour» du 3 février 1919 de la loge maçonnique «Droit et Justice» semble donner quelque consistance à cette hypothèse : «Considérant que l'impôt du sang largement et volontairement payé à la France et pour la plus noble des causes nous crée, s'il se peut encore, des liens plus étroits avec la Mère patrie et consacre notre assimilation entière et définitive avec nos frères de France $»^{50}$.

La droite martiniquaise a, longtemps, été l'héritière de cette conception du rapport à la France. Le temps a passé. La nature des liens a

\footnotetext{
${ }^{49}$ Cette formule signifie que les mulâtres réclament avec constance l'assujettissement au service militaire.

${ }^{50}$ In Historial Antillais, tome V, p.68. Il s'agissait de réagir à la rumeur selon laquelle la Martinique pourrait être cédée aux Etats-Unis.
} 
changé. Assurément, il y a encore beaucoup de Martiniquais qui considèrent la France comme la "Mère patrie ». Et, même, lorsque ce n'est pas le cas, l'histoire a tissé des liens que l'on ne peut ignorer. Personnalité composite ${ }^{51}$. Il n'est pas excessif de dire que tous les Martiniquais reconnaissent la composante française de leur personnalité. Mais ils la vivent différemment. Nombre d'entre eux considèrent que les autres composantes de leur personnalité doivent également être prises en compte. Ils sont Martiniquais et, pour certains justifient leur attachement à la France par des considérations économiques. Pour rendre compte de cette nouvelle «donne», un auteur américain, William Miles distingue au sein de la droite martiniquaise les «vieillards français» et les «chiffristes ${ }^{52}$. En d'autres termes, Miles considère qu'il y a deux fractions, les «nostalgiques », assimilationnistes, attachés au statut départemental et qui n'acceptent d'évolution statutaire/institutionnelle que si la même évolution intervient en France hexagonale; les "pragmatiques", attachés au niveau de vie que procure l'appartenance à la France et qui veulent en même temps que soit reconnue leur personnalité singulière. Ils ont voté « oui » à la consultation populaire du 7 décembre 2003. D'une certaine manière, pour eux, l'affirmation identitaire permet le développement. Cependant, l'avancée identitaire se fait progressivement et, comme pour la gauche, il ne s'agit pas d'une position dictée par une analyse objective permettant, dans le cadre du Désir de reconnaissance, d'affirmer la différence. La démarche n'est pas conquérante. Elle est défensive et se manifeste par la volonté d'abord timide (A) puis plus clairement affirmée de desserrer les liens avec la France (B).

\section{A - INSTILLER UNE DOSE DE DECENTRALISATION}

Deux périodes peuvent, à ce niveau, être distinguées. La première - pour un temps - avec Léon Laurent Valère. C'est, à vrai dire une amorce en forme d'étoile filante. La seconde, à peine moins rapide dans le temps, avec la tentative d'entrer en politique d'un homme d'affaires de couleur, Max Elizé.

\footnotetext{
${ }^{51}$ Analyse d'Edouard Glissant : «Il y a un peuple martiniquais mais qui ne se pense pas en tant que peuple. Il y a un peuple martiniquais qui est un peuple composite et non un peuple identitaire ». Antilla. N0 116. 10 novembre 2004.

${ }^{52}$ W. Miles. Paradoxe au Paradis: de la Politique à la Martinique. Paris : L'Harmattan, 1992.
} 


\section{L'AMORCE}

Elections cantonales de 1967. Un nouveau groupe départementaliste - donc de droite dans la terminologie habituelle - apparait, l'Union Démocratique Martiniquaise pour la promotion politique, économique et sociale de la Martinique dans l'ensemble national français. Ce mouvement prône une décentralisation administrative. Il a deux élus, deux avocats, Emmanuel Lucien et Léon Laurent Valère. Ce dernier est le chef de file du nouveau groupe qui se distingue par le fait qu'il n'est rattaché à aucun parti politique français. L'entourage de l'époque de Me Valère rapporte qu'il cherche à approcher Aimé Césaire. En vain. De fait, il est vite étiqueté comme l' «homme des békés $»^{53}$. Dès lors, le jeune avocat est perçu comme ayant partie liée avec les milieux d'affaires et son discours - modérément-décentralisateur ne sera pas entendu et il y renoncera sans insister.

\section{LA REVENDICATION ASSUMEE}

Une nouvelle étape est franchie avec l'intervention sur la scène politique de Max Elizé, ancien président de la Chambre de Commerce et d'Industrie de la Martinique. Il considère que la décentralisation doit être au service du développement et de l'affirmation identitaire. Max Elizé s'entoure, au début de la décennie 1980, de cadres martiniquais qu'il regroupe - peu de temps, il est vrai - au sein d'une association baptisée, l'ARUM ${ }^{54}$. Formés pour la plupart à l'économie et à la gestion mais aussi pour certains à la sociologie, le credo de ces cadres est l'appartenance à la République. Pour autant, ils considèrent que la centralisation est un frein au développement et que le fait d'être français ne signifie pas nécessairement pour un martiniquais de renoncer à sa personnalité singulière. Deux exemples nous permettrons, nous l'espérons, d'illustrer leur démarche reprise par un grand nombre de Martiniquais jusque là, pour la plupart, indifférents aux affaires publiques.

Le premier témoigne du refus de la dilapidation du patrimoine foncier.

\footnotetext{
${ }^{53}$ Dans le cadre de la campagne électorale, il tient à Didier, quartier huppé de Fort-de-France à l'époque plus encore qu'aujourd'hui, une réunion comme il le fait dans tous les quartiers du canton. Avec, cependant, une particularité. Elle a lieu au domicile de Raymond Cottrel, important béké du pays et on n' y accède que sur invitation.

${ }^{54}$ Nous ne sommes pas arrivé à trouver la déclinaison de cet acronyme.
} 
Le second, peut-être plus anecdotique, marque la volonté de voir reconnue la différence identitaire.

\section{- L'AFFAIRE ASATAHAMA}

Dans le courant de l'année 1974, il apparaît qu'une structure, vraisemblablement multinationale, nommée ASATAHAMA, ayant pour directeur général, le fils du député de la circonscription Sud de la Martinique a, dans cette région, le projet d'édifier un complexe touristique, gigantesque à l'échelle de la Martinique. Il s'agit de construire sur trois cents hectares (300 ha) au moins six (6) hôtels et mille deux cents (1200) villas. Le projet suscite l'indignation générale. Un Comité de Défense et de Sauvegarde des Salines et autres sites et plages de la Martinique est créé. Très vite, le comité présidé par un médecin, le docteur Hubert Montlouis qui n'est pas personnellement impliqué dans la vie politique revendique mille quatre cents (1400) adhérents. L'émotion est si vive que la presse nationale s'empare de l'affaire, le Préfet de la Martinique également et ce dernier adresse au Conseil général, le 28 septembre 1974, un rapport dans lequel il fait savoir que le directeur général d'ASATAHAMA renonce à son projet. Cette victoire a soudé une large fraction de la communauté martiniquaise et se fait jour l'idée que la protection de la terre participe de la défense de l'identité.

\section{- L'HEURE D'ETE}

Un décret du 17 octobre 1979 publié au Journal Officiel du 19 octobre 1979 indique que le passage à l'heure d'été est désormais applicable à la Martinique. Cette mesure considérée comme grotesque voire offensante mobilise, elle aussi, les Martiniquais au point qu'ils obtiennent son retrait.

Dans ces deux affaires, Max Elizé s'est nettement positionné comme un défenseur de l'identité. Il sera encore plus clair sur la question de la région monodépartementale. Il est clairement partisan de l'Assemblée Unique. Il s'exprime ainsi : "C'est une stupidité ! Ce n'est pas un choix ni de droite ni de gauche. C'est une idiotie $»^{55}$. La crudité du propos témoigne d'une véritable colère, Max Elizé étant réputé pour ses manières policées.

\footnotetext{
${ }^{55}$ Rapporté par Le Naif, Mars 1996, No 64.
} 
Mais comme Léon Laurent Valère, quelques années plus tôt, il n'arrive pas à éliminer Aimé Césaire de la vie politique et retourne à ses affaires ${ }^{56}$.

\section{A - CONCILIER LE RESPECT DE L' IDENTITE ET L'APPARTENANCE A LA FRANCE ET A L'UNION EUROPEENNE}

La première session du Congrès des élus départementaux et régionaux de la Martinique réuni les 21, 22, 23 février et 4 mars 2002 confirmée par la seconde session tenue, elle, le 10 avril 2003 a fait apparaitre clairement le clivage existant au sein de la droite et décrit sans nuances vingt ans plus tôt par William Miles ${ }^{57}$.

D'un coté, les «assimilationnistes fixistes », fortement campés sur leur position départementaliste et pour lesquels l'évolution ne peut intervenir que si elle se fait au même rythme qu'en France métropolitaine. Ils ont milité en faveur du «non» lors de la consultation populaire de décembre 2003, se démarquant, par conséquent, de la position sinon du gouvernement en tout cas de celle prêtée au Ministre de l'outre-mer de l'époque ${ }^{58}$. Adhérents de l'UMP, ils se sont un moment rapprochés de François Bayrou.

De l'autre, les «identitaires», pour la plupart réunis au sein du groupe «Osons oser». Ils ont plaidé pour le «oui», indiquant que l'évolution statutaire n'était pas, à leurs yeux, une fin en soi. Elle était seconde par rapport aux impératifs du développement économique et social. Présents aux élections régionales, ils n'ont obtenu aucun siège. A vrai dire leur discours se distinguait mal de celui des deux courants de la gauche «traditionnelle» et pas davantage de celui des «Patriotes». Aujourd'hui, certains d'entre eux et, en particulier leur chef de file Pierre Petit, semblent disposés à prendre de la distance par rapport à l'UMP: « Je n'ai pas

\footnotetext{
${ }^{56}$ Dans lesquelles il cherche à promouvoir, en le modernisant, le développement économique et ne cache pas que l'économie traditionnelle basée, à l'époque, sur la canne à sucre a vécu. Léon Laurent Valère a, lui, quitté la Martinique, renoncé au barreau et accompli une brillante carrière de magistrat qu'il a achevée comme Premier président d'une Cour d'Appel.

${ }^{57}$ Voir opus cit. p. 15.

${ }^{58}$ Il ne faut pas oublier que dès 1987, Jacques Chirac, alors Premier Ministre, déclarait à Fortde-France : « Notre ambition pour les Antilles doit être à la mesure des défis que nous avons à relever: l'intégration au grand marché européen, l'ouverture sur la zone caraïbe, la préservation de l'identité antillaise dans l'appartenance acceptée à la communauté nationale ». France-Antilles, 12 septembre 1987.
} 
d'inconvénient à être à l'UMP si ce parti me reconnaît comme parti politique »" Ils entendent mettre en place une structure politique martiniquaise qui « ne sera ni indépendantiste ni autonomiste ${ }^{60}$. Là aussi, la position a évolué « Osons, oser » s'est rangé derrière Nicolas Sarkozy.

A vrai dire, l'homme politique qui incarne ce courant novateur de ce qu'il est convenu d'appeler la droite martiniquaise est le Député-maire de Schoelcher, Alfred Almont. Ancien de l'équipe de Max Elizé, fervent admirateur d'Aimé Césaire, sa ville est très attachée au «devoir de mémoire » en même temps qu'à la coopération régionale. A cet égard, son action peut être comparée à celle du maire du Lamentin, Pierre Samot.

Droite et gauche ne se distinguent plus - si tel a jamais été le cas - par les critères traditionnels. Aujourd'hui, la prise en compte du facteur identitaire les rapproche. Même les partisans d'un alignement statutaire/institutionnel strict par rapport à la France l'intègre. Comment, alors, dans ce paysage politique renouvelé, envisager l'avenir?

\section{SCENARIOS POUR LE FUTUR}

Prévoir demain. Le pari est audacieux et l'entreprise peut paraître futile. Cependant, l'analyse du passé et du présent vaut surtout parce qu'elle est susceptible de fournir des indications permettant d'envisager ce que pourront être les grandes lignes des changements à intervenir. Encore faut-il qu'il ne s'agisse pas de spéculations gratuites mais que les hypothèses avancées prennent appui sur l'existant. Tel est l'objet de la prospective. Nous allons, donc, nous y essayer. En faisant trois observations préalables.

- L'approche de l'élection présidentielle peut constituer un élément de brouillage. Les uns et les autres vont se déclarer en faveur des candidats de leurs familles politiques métropolitaines d'origine qui ne sont d'ailleurs parfois que des « correspondants » avec l'objectif d'être bien placés au cas où leur champion l'emporterait. Du même coup, ceux qui sont favorables à une nouvelle consultation sur la question de la collectivité unique et de l'assemblée unique risquent d'y renoncer, au moins à brève échéance. En d'autres termes,

${ }^{59}$ France-Antilles, Lundi 20 février 2006, p.4.

${ }^{60}$ Ibidem. 
la «part française de l'identité » peut de nouveau prendre le dessus dans l'expression publique. Et l'évolution renvoyée aux calendes.

- La communauté martiniquaise existe. En ce qui nous concerne, le point n'est pas douteux même si la Constitution a préféré la notion de «population » à celle de «peuple». Dans la dernière période, la disparition de personnalités du monde artistique et culturel a été l'occasion de ressouder la communauté, profondément émue. Nous pensons ici à Loulou Boislaville, Edith Lefel, Henri Guedon. Cette émotion collective s'est manifestée également à l'occasion du crash aérien du 16 août 2005 qui a occasionné 152 victimes martiniquaises et a été ressenti comme un drame touchant l'ensemble des Martiniquais. Et que dire de l'exceptionnelle mobilisation pour obtenir l'abrogation de l'article 4 de la loi du 23 février 2005 ? Dans le même temps, sentant le groupe gravement en danger les Martiniquais commencent à se rassembler pour déterminer les moyens de faire face à la très inquiétante montée de la violence.

Communion passagère, dira-t-on, sans traces durables. Voire. Et s'il s'agissait plutôt de la phase de consolidation-accélérée ?-de la construction identitaire. Ne l'oublions pas. Pour un individu comme pour une collectivité, l'identité n'est pas un acquis. Elle se construit sur la durée, avec des ruptures et des continuités.

- L'Indépendance n'est pas à l'ordre du jour.

Sous le bénéfice de ces remarques, deux hypothèses principales peuvent être envisagées.

1. La poursuite dans la voie de la décentralisation acte II.

Nous sommes là dans la logique de la consultation populaire de décembre 2003. Le statut départemental est maintenu mais aussi la possibilité d'affirmer une certaine différence avec, par exemple, le droit à l'expérimentation. Le cas est d'autant plus vraisemblable que l'on voit mal ce qui, sur le fond, distingue la décentralisation telle qu'elle va se développer de ce que certains appellent l'Autonomie.

Il n'est, cependant, pas sûr que dans ce scénario les maux sérieux dont souffre la société martiniquaise puissent trouver un début de solution. 


\section{L'avancée progressive vers la souveraineté optimale.}

Aller dans cette direction implique que la communauté se fortifie. Ce qui, au plan politique, devrait se traduire par la constitution d'un Front Martiniquais qui aurait pour premier objectif d'obtenir l'application de l'article 74 de la Constitution et, par conséquent, un statut comparable à celui de la Polynésie. Les évolutions ultérieures étant toujours possibles au gré de la volonté populaire. Ce front qui pourrait être qualifié d'interclassiste regrouperait, faisant fi des divergences passées, l'ensemble des forces politiques, à l'exception, peut-être des «départementalistes fixistes » à moins qu'eux aussi ne se laissent entraîner par ce qui apparaîtrait comme un mouvement de fond.

Aujourd'hui, tel n'est pas le schéma qui prévaut. Le nouveau président du PPM, Serge Letchimy appelle, certes, à un vaste rassemblement pour obtenir l'Autonomie, mais ses premières démarches dans cette direction se sont soldées par un échec.

Pire, le sénateur Claude Lise, jusque là en «congé de parti », a créé une nouvelle organisation politique, «le Rassemblement Démocratique Martiniquais ». Il est prévu que le parti existerait, certes, mais que ses composantes bénéficieraient d'une très large marge d'action au plan territorial. Cette curieuse conception du parti - en tout cas en contradiction avec la

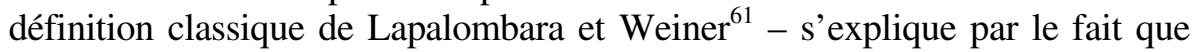
son épine dorsale est constituée par l'OPMI dont nous avons parlé précédemment, les «Divers Gauche ». Quant aux «dissidents » du PPM, il convient de noter que la «saignée » annoncée ne s'est pas encore manifestée. $\mathrm{Au}$ moins en ce qui concerne la représentativité des militants qui ont suivi le sénateur. Sauf erreur de notre part, deux maires de deux petites communes du Nord Caraïbe ont suivi le sénateur et une poignée de conseillers généraux ainsi qu'un petit nombre de conseillers municipaux de Fort-de-France.

A part Claude Lise, la seule personnalité politique venue du PPM, ayant quelque influence, est le premier vice- président du Conseil général, Claude Cayol qui aurait été un challenger dangereux pour le député Almont

${ }^{61}$ Lapalombara Joseph, Weiner Myron, Political parties and political development, Princeton University Press, 1966. 
dans la circonscription du Nord Caraïbe aux dernières législatives si son parti avait bien voulu l'investir.

L'affaiblissement annoncé du PPM n'est peut-être qu'une vue de l'esprit. Son image en souffrira, certes, mais au plan politique ?

Précisément au plan politique, il faut certainement attendre la constitution effective du nouveau parti pour cerner les différences entre les deux camps. Pour l'heure, elles n'apparaissent guère.

A supposer ces difficultés surmontées, ouvrant la voie à la constitution d'un Front Martiniquais, il est pertinent de se demander pour quelles raisons ce qui n'a pas été accepté en 2003, le serait demain. Peutêtre, pour la raison que mieux assurés d'eux-mêmes, les Martiniquais seraient apaisés quant à leur crainte d'un avenir fait de misère et prendraient conscience que la reconnaissance totale de leur identité singulière de même que la nécessité d'un développement économique que l'on aurait qualifié naguère d'autocentré suppose une réelle possibilité de décision.

La vraie difficulté n'est peut-être pas à ce niveau. Elle pourrait bien concerner la question du leader. Etant observé que, mis à part Serge Letchimy, les prétendants possibles sont, pour l'heure, tous septuagénaires ou à peu près. 


\begin{abstract}
RESUME
Du brouillage. Droite et gauche en Martinique. Quelle réalité ?

Dans les démocraties traditionnelles, la vie politique est généralement marquée par le clivage droite/gauche, même s'il est vrai qu'il a perdu beaucoup de sa pertinence, l'Allemagne en offrant un exemple typique. La distinction existe cependant. A la Martinique, département français ultra-périphérique, la situation se présente différemment. Longtemps la distinction entre droite et gauche s'est superposée à celle opposant les tenants du statu quo départemental et les partisans d'un changement de statut politique. D'un côté, à droite, les assimilationnistes se réclamant de la «mère patrie», de l'autre, à gauche, les «nationalistes» se positionnant dans la problématique de la souveraineté. Progressivement, les positions ont évolué. Sous l'effet de facteurs que l'article cherche à identifier, les assimilationnistes ont adopté une position plus identitaire et les nationalistes ont, de fait, renoncé au nationalisme politique au profit d'un «nationalisme culturel ». Cette situation s'est illustrée par des scrutins électoraux aux résultats apparemment contradictoires. A partir de cette nouvelle «donne », l'article se risque à envisager des scénarios pour le futur.
\end{abstract}

Mots-clés : autonomie, clivage droite/gauche, nationalisme, assimilationnisme.

\begin{abstract}
Interference. Right and left wings in Martinique. And the reality?

In traditional democracies, political life is generally marked by the left-right divide, even if it has indeed lost much of its pertinence, with Germany offering a typical example. The distinction does, however, exist. In Martinique, a French Overseas Departement, and European Outermost Region, the situation is presented in a different form. For quite some time, the distinction between right and left wings crossed that which opposed the maintaining of the status quo as a fulfledged French Departement and the supporters of a change in political status. On the one hand, to the right, the "Assimilationists" claim being part of the Motherland, and on the other hand, to the left, the "Nationalists" view themselves from a sovereignty perspective. Gradually, the positions evolved. Subject to the effects of factors which the article seeks to identify, the Assimilationists adopted a more identity-based stance and the Nationalists in fact abandoned political nationalism to adopt a "cultural nationalism". This situation was illustrated by a vote which yielded apparently contradictory results. Based on this new element, the article takes the risk of considering future scenarios.

Key Words: Autonomy, right-left divide, nationalism, assimilationism
\end{abstract}

